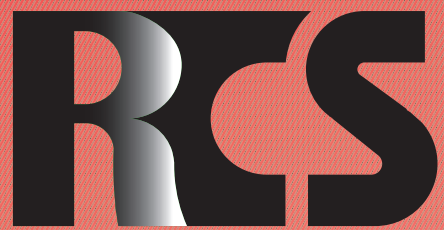

Depósito legal ppi $201502 Z U 4662$

Esta publicación científica en formato digital es continuidad de la revista impresa Depósito Legal: pp $197402 Z U 789$

- ISSN: 1315-9518 • ISSN-E: 2477-9431

Revista de Ciencias Sociales

Universidad del Zulia. Revista de la Facultad de Ciencias Económicas y Sociales Vol. XXVII. No. 2

Abril-Junio 2021

Esta publicación científica en formato digital es continuidad de la revista impresa Depósito Legal: pp $197402 Z$ Z789 ISSN: 1315-9518 

Rojas-Bahamón, M. (2021). Inteligencia artificial y condición humana: ¿Entidades contrapuestas o fuerzas complementarias?. Revista de Ciencias Sociales (Ve), XXVII(2), 502-513.

\title{
Inteligencia artificial y condición humana: ¿Entidades contrapuestas o fuerzas complementarias?
}

\author{
Arbeláez-Campillo, Diego Felipe* \\ Villasmil Espinoza, Jorge Jesús*** \\ Rojas-Bahamón, Magda Julissa***
}

\section{Resumen}

En el siglo XXI la inteligencia artificial se constituye en una fuerza que supera en muchos aspectos a la ficción, porque de cierto modo ya está presente en todos los ámbitos de la vida social, desde los motores de búsqueda por internet para determinar los gustos y preferencias en el acceso a la información digital, hasta en refrigerados inteligentes capaces de emitir órdenes de compra para mantener la disponibilidad de ciertos alimentos a medida que se van agotando. El objetivo de este ensayo es analizar las posibles problemáticas éticas, ontológicas y jurídicas que se derivan del uso generalizado de la inteligencia artificial en las sociedades actuales, como intento preliminar de resolver la interrogante planteada en el título. En lo metodológico se trata de un ensayo desarrollado mediante el uso de fuentes documentales escritas, como: Obras literarias, artículos de prensa internacional y artículos arbitrados publicados en revistas científicas. Se concluye, que la AI tienen el potencial para trastocar los estilos de vida de la civilización en general de muchas formas llegando, incluso, a alterar la condición humana de modo negativo al cambiar su identidad e integridad genética y debilitar el protagonismo de las personas en la construcción de sus propias realidades.

Palabras clave: Inteligencia artificial en el siglo XXI; significados de la condición humana; conflictos éticos, ontológicos y jurídicos; fuerzas complementarias.

\footnotetext{
Magister en Educación. Docente de la Universidad de la Amazonia, Colombia. E-mail: dfaca@hotmail.com iD ORCID: https://orcid.org/0000-0002-9041-9563

** Doctor en Ciencia Política con Postdoctorado en Derechos Humanos. Magíster Scientiarum en Historia de Venezuela. Licenciado en Educación, mención Historia. Profesor Titular de la Universidad del Zulia, Venezuela. E-mail: jvillasmil@fcjp.luz.edu.ve; jvillasmi152@hotmail.com (iD ORCID: https://orcid.org/0000-0003-0791-3331

*** $\quad$ PhD. en Educación y Cultura Ambiental. Profesora en la Institución Educativa Jorge Eliecer Gaitán, Universidad de la Amazonia, Colombia. E-mail: mjulissa@gmail.com (iD) ORCID: https://orcid.org/0000-0003-4882-1476
} 


\title{
Artificial intelligence and the human condition: ¿Opposing realities or complementary forces?
}

\begin{abstract}
In the21st century, artificial intelligence is a force that surpasses fiction in many respects, because in a way it is already present in all areas of social life, from internet search engines to determine tastes and preferences in access to digital information, to intelligent refrigerated ones capable of issuing purchase orders to maintain the availability of certain foods as they become exhausted. The objective of this essay is to analyze the possible ethical, ontological, and legal problems arising from the widespread use of artificial intelligence in today's societies, as a preliminary attempt to resolve the question raised in the title. Methodologically, it is an essay developed through the use of written documentary sources, such as: literary works, international press articles and arbitrate articles published in scientific journals. It is concluded that AI has the potential to disrupt the lifestyles of civilization in general in many ways, even altering the human condition in a negative way by changing its genetic identity and integrity and weakening people's prominence in building their own realities.
\end{abstract}

Keywords: Artificial intelligence in the 21st century; meanings of the human condition; ethical, ontological, and legal conflicts; complementary forces.

\section{Introducción}

¿Qué es la inteligencia? ¿Son realmente inteligentes los seres humanos? ¿Tiene la humanidad el monopolio de la inteligencia ante las otras formas de vida? ¿La vida se reduce únicamente a lo orgánico-biológico? Para poder contribuir asertivamente con el actual debate sobre la trascendencia y significación de la Inteligencia Artificial (IA) en el siglo XXI, se debe en principio intentar responder estas preguntas, destacando que la noción de inteligencia es por su propia esencia semántica: Polisémica y al mismo tiempo multidimensional, de ahí, que se habla cotidianamente de inteligencia cognitiva, emocional, conductual, inteligencias múltiples y artificial, entre otras. Convencionalmente:

La inteligencia es una capacidad mental muy general que implica habilidad para razonar, planificar, resolver problemas, pensar de forma abstracta, comprender ideas complejas, aprender con rapidez y aprender de la experiencia. (...) refleja una capacidad amplia y profunda para la comprensión del entorno, para ser capaz de capturar el significado de las cosas y darles un sentido, o para ingeniárselas a la hora de saber qué hacer. (Sanz, 2020, p.1)

Al decir, Ardilla (2010) sostiene que la inteligencia se muestra como la capacidad central para el ser, el hacer y el convivir en ámbitos tan diversos como: Los dominios lógico-matemáticos, las habilidades lingüísticas, el talento musical, lo intra e interpersonal y social, y, la dimensión corporal sistémico. Para la psicología y la neurociencia, se trata definitivamente de un fenómeno medible cuyo desarrollo o involución está asociada a factores filogenéticos, ambientales, educativos y culturales. En este orden de ideas, la noción de IA es hasta cierto punto subsidiaria de las teorías y conceptos sobre la inteligencia humana con la particularidad de que ahora:

(...) hace alusión concreta a un tipo particular de inteligencia tecnológica que, aunque tiene su punto de inicio en la 
persona, que es su artífice y causa primaria, puede funcionar con independencia $y$ autonomía frente a la misma, llegando incluso a superar en muchos aspectos las capacidades cognitivas y procedimentales de la humanidad. (Brito, Villavicencio y Sánchez, 2019, p.261)

Con todo, la noción de inteligencia ha sido reificada ${ }^{(1)}$ por un discurso antropocéntrico y logocéntrico -excesivamente acrítico- que postula tácitamente la supuesta superioridad intelectual de la humanidad, sin cuestionar sus paradojas, pero ¿hasta qué punto son inteligentes los sistemas políticos que se han producido y reproducido históricamente? Cuando están plagados de contradicciones autoritarias evidentes en las relaciones asimétricas de poder, que subordinan a la ciudadanía al acatamiento de los mandatos de los gobiernos, independientemente de lo absurdo que puedan resultar a veces ¿hasta qué punto son inteligentes los sistemas económicos existentes? Cuando depredan sistemáticamente los recursos naturales no renovables por mero afán de lucro y concentran la riqueza de forma exponencial en el 1\% de la población de los países desarrollados (Stiglitz, 2012; Parra, 2020).

Por ventura la IA artificial, pudiera en muchos aspectos superar las limitaciones y contradicciones de la inteligencia humana, profundizando su condición de ser una fuerza complementaria de la misma o, por el contrario, terminar resultando en un factor antagonista, el futuro lo dirá. Son precisamente estas ideas y otras similares, las que justificaron el desarrollo de este ensayo. Conviene recordar que, en muchas obras literarias o cinematográficas al estilo de Yo, Robot de Isaac Asimov o Terminator dirigida por James Cameron, la IA en su forma robótica-antropomórfica entra en conflicto con la humanidad en la misma medida que desarrolla altísimos niveles de autonomía que le permite a estas "entidades" desplegar un conjunto de decisiones que pueden resultar polémicas desde la perspectiva ética, ontológica o jurídica.

De cualquier modo, no cabe duda de que en el siglo XXI la IA se constituye en una realidad que supera en muchos aspectos a la ficción, porque está presente de una forma u otra en todos los ámbitos de la vida social moderna, desde los motores de búsqueda por internet para determinar los gustos y preferencias en el acceso a la información digital, hasta en refrigerados inteligentes capaces de emitir órdenes de compra para mantener la disponibilidad de ciertos alimentos a medida que se van agotando. Por lo tanto, el objetivo de este trabajo radica en analizar las posibles problemáticas éticas, ontológicas y jurídicas que se derivan del uso generalizado de la AI en las sociedades actuales, como intento de resolver la interrogante planteada en el título y como excusa para propiciar un debate al respecto sobre esta cuestión trascendental.

Por lo demás, este ensayo esta divido en 4 secciones particulares, pero lógicamente relacionadas. En el Estado del arte, se da cuenta de las obras selectas que sirvieron para modelar la concepción de la IA y sus distintas implicaciones para la condición humana. En la segunda sección, se explica el procedimiento metodológico que hizo posible la interpretación y organización de las fuentes consultadas, así como se aclara la postura epistémica de los investigadores. En el tercer apartado, interesó resolver el objetivo planteado, al menos de forma provisional para, en la última parte, arribar a las conclusiones y hallazgos del caso.

\section{Estado del arte}

De conformidad con los límites de extensión del género articulo científico, a continuación, se efectúa un breve balance de las diferentes obras y autores que apuntalaron con sus aportes el aparato teórico y analítico del ensayo y, simultáneamente, sirvieron de influencia para estructurar esta visión de la AI en el mundo de hoy, en tanto fenómeno tecnológico que, llegado el caso, puede contribuir significativamente en el proceso de transformación de la civilización humana en general de insospechadas maneras.

El trabajo de Vinuesa, et al. (2020), fue importante para visualizar la utilidad y 
versatilidad de IA en la sociedad moderna. En palabras de los autores, la IA adquiere un rol destacado en el impulso de las metas del desarrollo sostenible, mediante la implementación de algoritmos y software que, de contar con el respaldo de información cualitativa y cuantifica suficiente, pueden llegar a regular y supervisar el logro de más de un centenar de objetivos. No obstante, no se descarta que entre las limitaciones actuales de esta forma de inteligencia autónoma, resaltan ciertas lagunas en el manejo transparente de la data, errores de seguridad y conducción adecuada de los estándares éticos.

Del mismo modo, la investigación de André y Romy (2020) aportó luces en la elucidación de los requisitos previos de la inserción de IA en los procesos de enseñanzaaprendizaje en educación superior, contentivos al estudio de nuevos modelos de negocios en empresas de tecnología EdTech. A juicio de los autores la datificación de la realidad social da lugar a la emergencia de nuevos modelos de negocios, con impacto en el mercado y en la educación.

De ahí que, un número destacado de empresas de tecnología educativa, conocidas en sus siglas en inglés como (EdTech), intentan renovar los modelos educativos tradicionales mediante la implementación sistemática de la IA para el procesamiento de metadatos de distinta índole, provenientes del uso cotidiano de los motores de búsqueda de la web, entre otras fuentes, que dan cuenta de los gustos y preferencias de las personas. Concluyen que, la incertidumbre y la poca comprensión de datos estratégicos están frenando el desarrollo de soluciones y, en consecuencia, la IA es la mejor herramienta que se vislumbra para el avance de este tipo de negocios.

Por su parte, Belk, Humayun y Gopaldas (2020) indican que la IA no es necesariamente una idea novedosa tipica de la modernidad occidental, toda vez que ciertas civilizaciones antiguas intentaron representar a su modo, nociones similares a la IA mediante actividades mágicas y religiosas como la alquimia, llegando a estructurar una tradicion mítica en la cual la humanidad se siente fascinada o temerosa ante la impronta de otra forma de inteligencia alternativa que venga a cuestionar su hegemonia intelectual en el planeta o, a superar sus capacidades cognitica y procedimentales.

A modo de conclusion Belk, et al. (2020) señalan que, los enfoques de IA basados en sofware (suaves) o en la robotica (duros) pueden articularse en la persona humana desembocando en un proceso dificil de determinar a priori de transhumanismo. Por estas razones, urge desde ya la construccion consensuada de marcos ético, que orienten la acción y diseño de las políticas públicas para garantizar en todo momento la seguridad y resguardo de la dignidad humana ante el avance de la IA en todas las dimensiones de la realidad social. Por útimo, se valoran las dos tradiciones discursivas que se originan en torno a la IA: Por un lado, la visión escéptica y cautelosa y, por el otro, la mirada futurista que celebra el avance de esta forma de inteligencia sin mayor reparo al respecto.

Biagini, et al. (2020), destacan ademas, la autonomia de la IA para el manejo, en un futuro próximo, de los sistemas de energia como condición de posibilidad para la puesta en marcha de un parque energético sostenible, con muy poca supervisión humana. Los autores argumentan que la puesta en marcha de software y hardware de IA pueden impulsar asimismo una transición tecnológicamente evolutiva de la humanidad, a una fase superior de su ciclo vital, con abundante energía renovable producida y distribuida de forma racional por la IA.

Especial interés tuvo en el equipo de investigación, los trabajos del polémico historiador hebreo Yuval Noah Harari (2014; 2015), que vienen a confirmar ahora con evidencia empírica concreta las predicciones que en otro tiempo efectuó la ciencia ficción e, incluso, a rebasarlas en varios sentidos. Para él, la especie Homo sapiens se encuentra definitivamente en su ocaso y, como resultado de la conjugación de la ingeniería genética, la robótica y la IA se dará paso a otra entidad post-humana o supra-humana -según como se vea el fenómeno- al mismo tiempo bio- 
mecánica y cibernética, que vendría a superar las limitaciones materiales y cognitivas de la condición humana $\mathrm{y}$, paralelamente, a desarrollar sus habilidades y destrezas de modo exponencial. En este contexto próximo sostiene que:

La ingeniería cíborg irá un paso más allá y fusionará el cuerpo orgánico con dispositivos no orgánicos, como manos biónicas, ojos artificiales, o millones de nanorrobots, que navegarán por nuestro torrente sanguíneo, diagnosticarán problemas y repararán daños. Un cíborg de este tipo podrá gozar de capacidades que superarán con mucho las de cualquier cuerpo orgánico. (Harari, 2015, p.55)

\section{Igualmente, destaca:}

(...) un cíborg podría existir en numerosos lugares al mismo tiempo. Una doctora cíborg podría realizar operaciones quirúrgicas de emergencia en Tokio, en Chicago y en una estación espacial en Marte, sin salir de su despacho de Estocolmo. Solo necesitaría una conexión rápida a internet, $\mathrm{y}$ unos cuantos pares de ojos y manos biónicos. Pero, pensándolo bien, ¿por qué pares? ¿Por qué no cuartetos? De hecho, incluso estos son realmente superfluos. ¿Por qué un médico cíborg tendría que sostener en la mano un bisturí de cirujano cuando podría conectar su mente directamente al instrumento? (Harari, 2015, p.55)

No cabe duda que para criterios conservadores estas afirmaciones pueden resultar exageradas y fantásticas, sin embargo, una revisión, incluso superficial, de la historia del último siglo muestra que los vertiginosos avances científicos han logrado en la vida cotidiana cosas inimaginables para las generaciones de antaño, como las comunicaciones en tiempo real con personas de todo el mundo, que proporcianan los dispositivos moviles inteligentes con conexiones inhalabrincas a la internet de banda ancha; la socialización abierta del conocimiento científico, o las nuevas formas de trabajo, relaciones interpersonales y educación virtual que facilitan las Tecnologías de Información y Comunicación (TIC). Por este motivo, muy seguramente las hoy vanguardistas ideas de Harari sean superadas por el porvenir en las próximas décadas.

\section{Aclaratoria epistemológica y metodológica}

Los autores de este trabajo, suponen que pronto la humanidad experimentará una transformación del orden mundial vigente (Arbeláez-Campillo, Andreyevna y RojasBahamón, 2019; Villasmil, 2020), acontecida en parte por los efectos devastadores del nuevo coronavirus, así como por el agotamiento de los modelos políticos y económicos efectivos hasta el momento.

Como es lógico suponer, estas transformaciones venideras, impulsaran a su vez cambios paradigmáticos en la estructura de la ciencia, apuntalando lo que Martínez (2009) define como una nueva racionalidad, en tanto superación definitiva de los límites impuestos por el neopositivismo, al menos en los dominios de las ciencias sociales y humanas, como intento fructífero por revindicar sin prejuicios saberes humanísticos como la filosofía, la literatura y el arte, en el marco de una concepción sistémica unificada del conocimiento científico que, sin perder rigurosidad en su metódica y cuerpos teóricos es simultáneamente: Objetivasubjetiva, inductiva pero profundamente interdisciplinaria; quizá rememorando la integridad y el sentido holístico del conocer que identificó a los antiguos o los renacentistas.

Es precisamente, animados por estas posturas gnoseológicas que presagian revoluciones paradigmáticas, donde emerge con fuerza el ensayo, no solo como un género literario de ruptura que destaca:

(...) por su libertad formal y temática, sino también por su ideología -en tanto su advenimiento se produjo en gran medida como reacción hostil hacia los géneros establecidos-, el ensayo es un caso ejemplar de visibilidad de la problemática genérica. Es un caso límite, cuando no un punto ciego, en cualquier toma de posición del pensamiento literario, que desde Aristóteles hasta nuestros días se 
ha sostenido sobre los pilares de la teoría de los géneros. (Camprubí, 2014, p.101)

(negritas añadidas)

En efecto, el ensayo crítico es también una herramienta clave del discurso científico en general, que permite el decurso de una hermenéutica libre con cierta originalidad, con arreglo a la combinación de factores como: La creatividad de los autores, criticidad y flexibilidad en el manejo de las fuentes, más allá de los estándares formales rígidos característicos de las monografías tradicionales que reproducen la subordinación cognitiva a los paradigmas dominantes, al tiempo que de cierto modo obliteran las innovaciones.

En términos operativos, en un primer momento se procedió a la delimitación del tema en reconocimiento de la trascendencia de la IA para el mundo actual y venidero. Seguidamente, se efectuó un arqueo de fuentes documentales escritas, en formato físico y digital, conformada por artículos científicos ubicados en reconocidas bases de datos: Scopus, WOS y Dialnet, entro otras; obras literarias y artículos de prensa internacional, que más allá de sus diferencias de textualidad y género, coinciden en otorgar una centralidad inusitada a la IA, en su varias formas y modalidades de existencia. Por último, se procedió a redactar el trabajo de conformidad con la normativa para autores de la Revista de Ciencias Sociales, para su evaluación, contrastación y, si procedía, publicación.

\section{Inteligencia artificial y condición humana: Problemáticas éticas, ontológicas y jurídicas que derivan de su uso generalizado en la sociedad}

Cuando se debaten las grandes posibilidades que derivan de la propagación generalizada de la IA en la sociedad, rápidamente se pueden observar al menos dos posiciones encontradas sobre este fenómeno: Por un lado, se revelan las personas e investigadores que suponen en esta forma de inteligencia una herramienta multidimensional para el mejoramiento de las condiciones de vida de la humanidad, al adelantar trabajos, procesos y tomar decisiones de forma autónoma y eficaz en función de los intereses y necesidades de las personas situadas en su contexto diferencial. Por el otro, no faltan voces críticas que presagian en la IA la posibilidad de alterar, para mal, el curso histórico de las sociedades en el siglo XXI, sin por ello, incurrir en anacronismos o posturas retrogradas.

En este hilo conductor, $-\mathrm{y}$ sin caer en una falacia de falso dilema- toda reflexión sobre el tema implica un cierto posicionamiento en torno a las posturas señaladas y este ensayo no es la excepción. Sea como sea, la IA ha demostrado hasta el momento una significativa versatilidad para coadyuvar a la gestión de casi todas las actividades humanas como: $\mathrm{La}$ educación, la salud, las finanzas, la recreación, las labores de limpieza del hogar y hasta en el desempeño de algunas profesiones liberales, en las que prevalece la técnica, el conocimiento intelectual, la creatividad y la experiencia. Hoy en día, por ejemplo, aplicaciones disponibles en teléfonos inteligentes como: ADA, iDoctus y Endomondo basadas en IA pueden desarrollar un acertado diagnóstico médico de diversas patologías, mientras que otras como: The Law Guide o SmartLeges hacen lo mismo en materia jurídica.

No obstante, científicos como Stephen Hawking (BBC News, 2014) no dudaron en su momento en augurar los peligros de la IA para la raza humana, argumentando que: "Los humanos, que son seres limitados por su lenta evolución biológica, no podrán competir con las máquinas, y serán superados" (p.1). Según el extinto físico británico una completa IA podría además auto-diseñarse con unos propósitos que, según su rápida evolución, podrían superar todos los controles antrópicos e insurgir contra las personas, al tiempo que no negó el potencial de este tipo de inteligencia para resolver gran variedad de problemas (BBC News, 2014).

Previendo esta situación de conflicto entra la robótica como máxima manifestación material de la IA y, la condición humana, el célebre escritor de ciencia ficción Isaac Asimov 
ya en una época temprana había formulado sus tres reglas fundamentales de la robótica:

1. Un robot no hará daño a un ser humano o, por inacción, permitirá que un ser humano sufra daño. 2. Un robot debe hacer o realizar las órdenes dadas por los seres humanos, excepto si estas órdenes entran en conflicto con la $1^{\circ}$ ley. 3. Un robot debe proteger su propia existencia en la medida en que esta protección no entre en conflicto con la $1^{\mathrm{a}}$ o la $2^{\mathrm{a}}$ ley. (Asimov, 1942, p.13)

De la lectura entre líneas de estas reglas o leyes que emergen del cuento corto Runaround publicado originalmente en 1942 , se infiere que el destino de todo robot es subordinar sus actuaciones a las órdenes dadas por los seres humanos y velar, por lo tanto, porque las personas no sufran ningún daño a costa incluso de su propia existencia, bajo la premisa categórica No. 1.

Por lo demás, queda claro que Asimov plantea el inicio del debate ético que buscaba regular desde los dominios de la ficción literaria el ejercicio de la praxis de la IA para evitar todo conflicto o tensión de estas entidades nacientes con la humanidad en su conjunto. De hecho, esta preocupación ética ocupara un lugar prominente en sus principales obras: Yo, Robot (1950); Hombre bicentenario (1976) y la saga Fundación ${ }^{(2)}$.

Sin ninguna duda la IA, en todas sus expresiones y modalidades es susceptible a tratamiento ético, por los riesgos y posibilidades que implica su propia existencia que está en desarrollo constante, mucho más cuanto que, todo indica que se trata de una entidad que evoluciona dialécticamente, tiene la capacidad de actuar con autonomía y de aprender y auto-modelarse con base a su propia experiencia individual y colectiva a un ritmo que rebasa las capacidades humanas. De hecho, círculos selectos como el Club Bilderberg incluye en su agenda de debates internacionales el paradigma de la IA como fuerza transformadora de las sociedades modernas (Esparza, 2015), lo que ha generado un conjunto de teorías conspirativas en las redes sociales.

En este punto crucial conviene aclara que la reflexión ética, siguiendo a Savater (1999), es la preocupación filosófica que entraña el ejercicio de la libertad individual que opta por la búsqueda del buen vivir, con todo lo que ello representa desde la perspectiva moral. De igual modo, la visión de las problemáticas que surgen del ejercicio de la libertad es subsidiaria de la ética humanística de Fromm (2003), para quien toda reflexión ética se sustenta además, en un criterio formal y material que vincula la praxis de las personas concretas con preceptos morales generales o particulares, utilitarios o dogmáticos, con base al principio de que es la conciencia del hombre -por sí sola- la que puede determinar el criterio de la virtud o vicio de sus actuaciones y no necesariamente una autoridad que lo transciende, lo que apuesta por la promoción de la autodeterminación de la voluntad sin la necesidad de fuerzas coercitivas externas a la misma, como el Estado o la religión.

En ambos casos, Savater (1999); y Fromm (2003) consideraran tácitamente que la persona humana es, en esencia y existencia, el único ser dotado de conciencia y voluntad capaz de actuar con libertad y, en consecuencia, que justifica un tratamiento ético. A pesar de estas posturas antropocéntricas que han regido hasta ahora la materia, el desarrollo de la IA cuestiona el paradigma dominante para el cual la inteligencia es un atributo exclusivamente humano y la vida se reduce únicamente a su esencia biológica, lo que posibilita al menos teóricamente un debate ético post-humano con mucho más sentido en las décadas venideras, en la misma medida que se disipen todas las dudas sobre la voluntad y conciencia particular de la IA, para ser y hacer con base a las condiciones del hardware y software que determinan su propia existencia y su fuerza autopoiética.

Sin más dilaciones ¿Cuáles serían desde ya las principales problemáticas éticas que pueden surgir directa o indirectamente de la propagación de la IA a todas las dimensiones de la vida social?, en este punto conviene recordar que, así como en su momento la primera revolución industrial significó la perdida masiva de empleos al sustituir hombres 
por maquinas, más baratas y eficientes, muy seguramente en las próximas revoluciones telemáticas y nanorrobótica que tendrán como factor protagónico a la IA, la situación no será muy diferente a lo acontecido en el siglo de las luces y los resultados en este sentido serán más dramáticos, toda vez que estas entidades tienen usos múltiples en casi todos los ámbitos imaginables.

En el escenario descrito, se está ante un evento que altera materialmente el principio de la ratio política, tal como lo entiende Duseel (2001), porque un nuevo orden mundial dominado por la IA, en cuanto razón práctica material no solo no se ocuparía de la condiciones que determina la producción y reproducción de la vida buena para los humanos, sino que además, introduce ahora una fuerza externa a la humanidad en procesos clave para el mantenimiento del equilibrio del ecosistema político y social, como la producción de bienes y servicios, la distribuciones de bienes de valores y la división social del trabajo y del conocimiento.

Pudiera argumentarse, que la impronta de la IA traería consigo la reducción de las jornadas laborales para beneficio de personas y comunidades enteras, incrementando paralelamente los espacios de recreación, convivencia familiar y formación, y que, igualmente, las pérdidas de potenciales empleos cooptados por maquinas inteligentes será subsanada por una política pública que garantizaría un ingreso básico general para todas las personas, similar a las experiencias de la seguridad social en el marco del Estado de bienestar posteriores a la segunda guerra mundial. No obstante, toda la evidencia histórica demuestra que en el sur global estas políticas siempre han sido insuficientes y limitadas y no garantizarían en ningún caso los recursos necesarios para el logro de una vida por encima de la pobreza y la precariedad, argumentar en sentido contrario sería ilusorio o demagógico.

Si a esto se le suma, que probablemente los grandes beneficios de la IA serán monopolizados por la elites políticas y económicas tal como sucedió en América Latina con los distintos programas modernizadores, que aun hoy han sido incapaces, según Morales, Villasmil y Martínez (2019), de aportar soluciones definitivas a la pobreza, la inequidad y las falta de oportunidades que tienen las grandes mayorías en la región para apuntalar su desarrollo, entonces la IA vendría a incrementar más bien las desigualdades y los mecanismos de exclusión social porque dotaría a unos pocos de un conjunto de ventajas relativas, en detrimento de los que no tengan acceso al disfrute de estas tecnologías.

También, la IA potenciara aún más los mecanismos de control social formales e informales de gobiernos autoritarios, para deterioro de los espacios de participación democrática y liderazgo ciudadano logrados históricamente mediante arduas luchas por todo el orbe. Si resulta exagerada esta afirmación basta examinar la forma como ha funcionado hasta ahora en China la red social conocida como Sistema de Crédito Social, que combina software de reconocimiento facial, geolocalización en tiempo real e IA, para premiar o castigar a las personas con una escala de puntuación que puede, en ciertos casos donde se atenta contra "la pureza ideológica del sistema" desembocar en la prohibición de salir del país, viajar en trenes u optar por un crédito bancario (Garrido y Shanghai, 2019).

Como epifenómeno de la cuestión ética, se visualiza asimismo una serie de problemáticas ontológicas que interesa revisar a grosso modo. La ontología, se refiere en este caso a la búsqueda del sentido último que define al ser humano en su dignidad intrínseca. Desde este punto de vista, el ser o la persona humanos ha construido históricamente su condición de ser, no solo como resultado de los imperativos categóricos de su biología, sino muy especialmente, del accionar intersubjetivo de atributos como la inteligencia, la voluntad, la capacidad de cooperar que caracteriza a la especie y la cultura, en tanto fuerza de reorganización constante de la naturaleza.

En efecto, los principales problemas ontológicos que derivan del uso general de la IA en el mundo de hoy, son aquellos que se modulan por su efecto primario para 
transformar al ser humano en el futuro en una entidad diferente, que no se puede determinar a plenitud en sus características y ethos esenciales en este momento. Si Harari (2015), tiene razón en su visión prospectiva, la especie Homo experimenta desde ya su ocaso biológico y material, fenómeno que trae consigo más preguntas que respuestas: ¿La fusión de IA con la humanidad subvierte la condición humana? ¿Cuáles son los límites éticos de todo proceso evolutivo? ¿El nuevo ser que surge de la combinación de la ingeniería genética, la nanorrobótica y la IA es necesariamente inaceptable? Ya en las postrimerías del siglo XIX, el gran pensador alemán Friedrich Nietzsche sentenció que:

El hombre sólo existe para ser superado. «El hombre es una cuerda tendida entre la bestia y el superhombre -una cuerda sobre un abismo-». De ahí que su grandeza esté en el hecho de «ser un puente y no una meta» y que lo que en él cabe amar sea «que es una transición y un ocaso». (Nietzsche, 2003, p.499)

Quizá el desarrollo de la tecnología en general y de la IA en particular, sea la potencia que impulse la superación definitiva de la persona humana, tal como se ha conocido ontológicamente hasta ahora. O tal vez, esta superación integral del Homo sapiens coincida con lo que Nietzsche imagino como el transito de la bestia al superhombre $y$, de paso, a una compleja encrucijada en la cual se debate el ocaso de la humanidad o la evolución de sus capacidades a niveles propios de los dioses, tal como supone Harari (2014).

A ciencia cierta, no hay nada seguro y solo se puede especular al respecto, pero en cualquier escenario las transformaciones que se materializaran en las décadas venideras no serán fáciles y traen aparejados cambios en la identidad de las personas pudiendo transformar su integridad genética $\mathrm{y}$, por 10 tanto, alterar significativamente su ser, hacer y convivir junto a la forma como sienten y viven sus mundos de vida, que está fijada en todo momento por las limitaciones y subjetividades propias de la condición humana en esta fase de su desarrollo histórico.

Por último y no menos importante, la IA requiere en cada momento de la reproducción de un conjunto leyes que colocan a prueba a los saberes jurídicos en su capacidad para normatizar y regular en el presente y futuro, el diseño, producción y uso de la IA. En este punto, Leret (2005) destaca la íntima relación existente entre biotecnología, derecho y bioética, que se da ante el acelerado desarrollo de la tecnología en los dominios de la informática, medicina y la genética, para resguardo de la identidad de las personas y de su integridad e identidad genética.

De nuevo en este punto, surgen preguntas legítimas como: ¿Una persona confeccionada genéticamente para lograr ciertos atributos genotípicos y fenotípicos contraviene el decurso evolutivo natural de la especie?, ¿estos avances dividirán en dos grupos a la humanidad: las personas "normales" y los superdotados por manipulación genética y/o implantes biomecánicos? Aunque no hay repuestas definitivas al respecto, queda claro desde ya, que serán las naciones desarrolladas las más favorecidas por estas mejoras, lo que reforzará aún más la brecha tecnológica y científica que hay entre el norte y el sur.

Desde el advenimiento del programa filosófico de la modernidad política, al decir de Calvano (2017; 2018), subyace en los sistemas jurídicos de occidente una idea de justicia, democracia y equidad que, mediante dispositivos simbólicos y conceptuales como: El estado de derecho y la dignidad humana, buscan proteger a las personas comunes de situaciones que vulneren su ethos o deterioren sus condiciones de vida. En este sentido, la producción de los cuerpos normativos que se generen para regular la IA deberá centrarse en por lo menos 4 aspectos cruciales, que se visualizan desde ya:

a. Desarrollar consensuadamente un marco legislativo internacional que subordine el diseño, producción y desarrollo de la IA a la dignificación de la vida en su tipología biológica. Tal como postula la primera ley de la robótica de Asimov.

b. Fijar los principios éticos y axiológicos universales que den sentido y propósito a la IA como una herramienta al servicio del 
desarrollo de las capacidades humanas, tal como las entiende Nussbaum (2012), es decir, como posibilidades inconmensurables de ser $\mathrm{y}$ hacer en el marco de un proyecto de vida, elaborado desde la soberanía individual.

c. Penalizar la instrumentalización de la condición humana por la fusión de implantes tecnológicos de IA, que transformen la conciencia y/o obliteren el criterio ético de las personas para ser y hacer libremente.

d. Castigar el uso de la IA como dispositivo de control social formal e informal para apuntalar un ordenamiento totalitario, al estilo de las ficciones distópicas como Black Mirror, Un Mundo Feliz de Aldous Huxley o 1984, de George Orwell.

El éxito de una agenda legislativa de esta naturaleza dependerá no solo de la voluntad política de gobiernos de avanzada para hacer un buen uso de la IA, sino, además, de la presión que haga la sociedad civil organizada para defender sus derechos en una democracia sustantiva para la cual sería inaceptable que IA se tornará paulatinamente en un dispositivo de dominación, independientemente de sus múltiples posibilidades de desempeño presentes en toda clase de dispositivos tecnológicos.

\section{Conclusiones}

La IA y la condición humana se debate inexorablemente en ser ¿entidades contrapuestas o fuerzas complementarias? Esencialmente todo dependerá del uso general que las personas hagan de esta tecnología, de los propósitos con que sea diseñada y de los resultados concretos que se obtengan de la misma para beneficio o deterioro de la vida en general, claro está, si es que la humanidad puede mantener controlada a esta forma de inteligencia bajo ciertos estándares éticos y bioéticos en el triángulo que conjuga IA, robótica e ingeniería genética. Hasta ahora, aparentemente la IA pudiera en muchos aspectos superar las limitaciones y contradicciones de la inteligencia humana, profundizando su condición de ser una fuerza complementaria de la misma.

No obstante, no debe descartarse a priori que en cierto momento de su evolución la IA en sus variadas modalidades de existencia, entre en conflicto con la humanidad en la misma medida que desarrolla altísimos niveles de autonomía que le permiten desplegar un conjunto de decisiones que pueden resultar polémicas desde la perspectiva ética, ontológica o jurídica. Bien sea como resultado de su propia voluntad consciente o, por su uso como herramienta de control y dominación social, como ya está sucediendo en países como China, donde la IA está al servicio de un ordenamiento autocrático.

Por las razones aludidas a lo largo del texto, ante las dos posiciones argumentativas sobre la IA que la visualizan: Por un lado, como una herramienta para el mejoramiento de personas y colectividades de cara al futuro $o$, por el otro, como una fuerza que debe ser analizada críticamente por las distintas amenazas que directa o indirectamente devienen de su uso, la opción se inclina por esta segunda postura sin desmeritar por ello sus múltiples contribuciones a la vida moderna. Todo indica que, en el nuevo orden mundial que se está estructurando la IA ocupará un rol central que bien podría augurar el transito del hombre a una entidad cualitativamente superior o, por el contrario, a la erosión moral de la condición humana. Desde esta perspectiva, hay más preguntas que respuestas y buenos motivos para ser cautos ante lo que se avecina en esta etapa de transición global.

Por lo demás, IA es un tema tan amplio y complejo que demanda en todo momento de un estudio interdisciplinario que la aborde en sus variadas dimensiones, como condición de posibilidad para comprender el fenómeno en su totalidad dialéctica. Con este propósito, no es descabellado proponer más y mejores líneas de investigación que den cuenta en la teoría y en la realidad, sobre las problemáticas éticas, ontológicas, jurídicas, políticas, psicológicas y antropológicas, que se derivan del cada vez más generalizado uso de esta tecnología. 
Arbeláez-Campillo, Diego Felipe; Villasmil Espinoza, Jorge Jesús y Rojas-Bahamón, Magda Julissa

Inteligencia artificial y condición humana: ¿Entidades contrapuestas o fuerzas

complementarias?

\section{Notas}

${ }^{1}$ Al decir de Ritzer (1999): "La reificación puede considerarse como el proceso por el que se llaga a creer que las formas sociales humanamente creadas son naturales, universales y absolutas y, consecuentemente, que esas formas sociales adquieren, de hecho, esas características. El concepto de reificación implica el convencimiento de las personas de que las estructuras sociales están fuera de su control y son inalterables" (p.191). Aunque pudiera argumentarse que la inteligencia humana tiene una base biológica neuronal, no es cierto que sea un atributo exclusivamente humano. A nuestro entender la inteligencia en un concepto de base social que varía en el tiempo y la cultura. Por lo demás, todas las formas de vida poseen cierta inteligencia en función de las características de su especie que les permite adaptarse al medio y sobrevivir inclusive en ambiente extremos como los llamados organismos extremófilos, de ahí que suponemos que la noción de inteligencia ha sido reificada.

${ }^{2}$ Para una revisión de las principales obras de Asimov se recomienda consultar: https:// www.europapress.es/cultura/libros-00132/ noticia-24-anos-isaac-asimov-10-novelas fundamentales-20160406165615.html

\section{Referencias bibliográficas}

André, R., y Romy, H. (2020). Prerequisites for artificial intelligence in further education: identification of drivers barriers, and business models of educational technology companies. International Journal of Educational Technology in Higher Education, 17, art. 14. https://doi.org/10.1186/ s41239-020-00193-3

Arbeláez-Campillo, D. F., Andreyevna, M., y Rojas-Bahamón, M. J. (2019). Las pandemias como factor perturbador del orden geopolítico en el mundo globalizado. Cuestiones Políticas,
36(63),134-150.

Ardilla, R. (2010). Inteligencia. ¿Qué sabemos y qué nos falta por investigar? Revista de la Academia Colombiana de Ciencias Exactas, Fisicas y Naturales, 35(134), 97-103.

Asimov, I. (1942). Runaround. Street \& Smith.

BBC News (02 de diciembre de 2014). Stephen Hawking: "La inteligencia artificial augura el fin de la raza humana". $B B C$ News. https://www.bbc.com/mundo/ ultimas noticias/2014/12/141202 ultnot hawking inteligencia artificial_riesgo humanidad_egn

Belk, R., Humayun, M., y Gopaldas, A. (2020). Artificial Life. Jounal of Macromarketing, 40(2), 221-236. https://doi. org/10.1177/0276146719897361

Biagini, V., Subasic, M., Oudalov, A., y Kreusel, J. (2020). The autonomous grid: Automation, intelligence and the future of power systems (Short Survey). Enerdy Research and Social Science, (65), 122-139.

Brito, P., Villavicencio, C., y Sánchez, P. (2019). Reflexiones sobre posibles conflictos entre la inteligencia artificial y el futuro de la sociedad. Revista de la Universidad del Zulia, 10(28), 260280.

Calvano, L. (2017). Modernidad política: Miradas y significados. Revista Cuestiones Politícas, 33(58), 37-48.

Calvano, L. (2018). Contrato social y modernidad política en Colombia. Fondo Editorial de la Universidad Rafael María Baralt (UNERMB).

Camprubí, C. B. (2014). El Ensayo en la Teoría de los Géneros. Castilla. Estudios de Literatura, (05),101-123.

Dussel, E. (2001). Hacia una filosofia política critica. Derechos humanos y desarrollo. Desclée. 
Esparza, R. (12 de junio de 2015). El Club Bilderberg lanza su nuevo paradigma: La inteligencia artificial. Hispanidad. https://www.hispanidad.com/ confidencial/el-club-bilderberg-lanzaun-nuevo-paradigma-la-inteligenciaartificial 212299 102.html

Fromm, E. (2003). Ética y psicoanálisis. Fondo de Cultura Económica.

Garrido, L., y Shanghai, J. (20 de octubre de 2019). Menos libertad vs más desarrollo económico. Buen ciudadano, a la fuerza: China acelera su plan de control social para 2020. El Confidencial https://www.elconfidencial.com/ mundo/2019-09-20/buen-ciudadanoa-la-fuerza-china-acelera-su-plan-decontrol-social-para-2020_2201731/

Harari, Y. N. (2014). De animales a dioses. Una breve historia de la humanidad. Debate.

Harari, Y. N. (2015). Homo Deus: Breve historia del mañana. Debate.

Leret, M. G. (2005). Derecho, biotecnología y bioética. Ediciones CEC.

Martínez, M. (2009). La nueva ciencia: Su desafio, lógica y método. Trillas.

Morales, Y. R., Villasmil, J. J., y Martínez, R. D. (2019). Democracia a la palestra: ¿Gobierno delpueblo o degeneración del poder? Revista de Ciencias Sociales (Ve), XXV(E-1), 236-252. https://doi. org/10.31876/rcs.v25i1.29611

Nietzsche, F. (2003). Obras inmortales II
Tomo 2. Tecnos Editorial, S.A.

Nussbaum, M. (2012). Crear capaciades: Propuesta para el desarrollo humano. Editorial Paidós.

Parra, R. (2020). Una perspectiva del mundo que se nos avecina. Revista de la Universidad del Zulia, 11(29), 3-5. https://doi.org/10.46925/rdluz.29.01

Ritzer, G. (1999). Teoría sociológica clásica. McGraw-Hill/ Interamericana de España.

Sanz, C. (12 de marzo de 2020). ¿Qué es la inteligencia? eMS El mundo del superdotado. https://www. elmundodelsuperdotado.com/que-esla-inteligencia/.

Savater, F. (1999). Ética para Amador. Ariel.

Stiglitz, J. E. (2012). El precio de la desigualdad: El 1\% de la población tiene lo que el 99\% necesita. Taurus.

Vinuesa, R., Azizpour, H., Leite, I., Balaam, M., Dignum, V., Domisch, S., Felländer, A., Langhans, S. D., Tegmark, M., y Nerini, F. F. (2020). The role of artificial intelligence in achieving the Sustainable Development Goals. Nature Communications, 11, art. 233. https://doi.org/10.1038/s41467-01914108-y

Villasmil, J. (2020). La fragilidad de las civilizaciones humanas. Cuestiones Politicas, 37(64), 10-14. 